\title{
Cerebral vasospasms following endoscopic endonasal surgery for pituitary adenoma resection in the absence of post-operative subarachnoid hemorrhage
}

\begin{abstract}
The endoscopic endonasal approach (EEA) is a widely accepted and commonly utilized approach for the resection of various pituitary tumors. While complications commonly include diabetes insipidus, cerebrospinal fluid leaks, and anterior lobe insufficiency cerebral vasospasm may also rarely occur. Herein, we report the unique case of a 44-year-old female who underwent uncomplicated endoscopic endonasal surgery for resection of a giant pituitary adenoma. Subsequent cerebral vasospasms were identified on postoperative day 3 and 19 resulting in ischemic strokes with neurological consequence. In the postoperative period, imaging at no point revealed any evidence of subarachnoid hemorrhage or hematoma formation in the subarachnoid space. Risk factors for cerebral vasospasm are discussed and the potential for subsequent vasospasm events is addressed.
\end{abstract}

Keywords: stroke, neurosurgery, subarachnoid hemorrhage, pituitary adenoma, vasospasm, endoscopic endonasal surgery
Volume 4 Issue 3 - 2016

\author{
Paul S Page,' Daniel D Kim,' Graham C \\ Hall, ${ }^{2}$ Maria Koutourousiou ${ }^{2}$ \\ 'University of Louisville School of Medicine, Louisville, USA \\ ${ }^{2}$ Department of Neurosurgery, University of Louisville, \\ Louisville, USA
}

Correspondence: Maria Koutourousiou, 220 Abraham Flexner Way, Suite 1500, Department of Neurosurgery, University of Louisville, Louisville, KY 40202, USA, Tel 502-582-7624, Fax 502582-7477, Email maria.koutourousiou@louisville.edu

Received: August 07, 2015 | Published: March 08, 2016
Abbreviations: EES, endoscopic endonasal surgery; CSF: cerebral spinal fluid; SAH, subarachnoid hemorrhage; aSAH, aneurysmal subarachnoid hemorrhage; MRI, magnetic resonance imaging; EEA, endoscopic endonasal approach; BMI, body mass index; ECG, electrocardiogram; CTA, computed tomography angiography; CT, computed tomography; IVH, intraventricular hemorrhage; FSH, follicle stimulating hormone; LH, luteinizing hormone; TSH, thyroid stimulating hormone; $\mathrm{GH}$, growth hormone; $\mathrm{ACTH}$, adrenocorticotropin hormone; TCD, transcranial doppler; EVD, extraventricular drain; GCS, glasgow coma scale (GCS)

\section{Introduction}

Pituitary adenomas are among the most common primary intracranial tumors in adults occurring in up to $15 \%$ of pituitary glands observed at autopsy and 20\% of radiological exams. ${ }^{1}$ Endoscopic endonasal surgery (EES) is a commonly utilized and generally welltolerated technique for the transsphenoidal removal of such tumors. Despite its widespread practice EES for pituitary resection is not without its complications. Commonly encountered complications include diabetes insipidus (7.0\%), epistaxis $(1.7 \%)$, anterior lobe insufficiency $(1.3 \%)$, or cerebral spinal fluid leakage $(0.6 \%){ }^{2}$ In addition to these complications, less common complications such as cerebrovascular vasospasm and hemorrhagic stroke secondary to vascular damage have been rarely reported. ${ }^{3,4}$

Cerebral vasospasm is a well-documented complication of subarachnoid hemorrhage (SAH) occurring in $67 \%$ of such cases. ${ }^{5}$ Symptoms may present as a variety of neurological changes occurring as early as a few hours to 13 days after hemorrhage onset. ${ }^{6}$ Despite advances in management, it is reported that $25 \%-50 \%$ of vasospasm survivors demonstrate at least some residual disability and death may occur in up to $23 \%{ }^{7,8}$ Though the exact mechanism of vasospasm is unclear, vascular inflammation, resulting from extravascular clot formation, is believed to be intimately involved in addition to genetic influences and dysfunctional autoregulation.
In addition to cases of $\mathrm{SAH}$, vasospasm has also been reported, though rarely, as a potential complication of pituitary adenoma resection. Cases of vasospasm after pituitary resection have been reported in a variety of surgical approaches including transsphenoidal, transcranial, fronto-temporal, and frontal approaches. ${ }^{9-11}$ While the exact etiology is unknown, it is suspected that mechanical manipulation of the vasculature, irritation by introduced packing materials, release of vasoactive mediators from the hypothalamus, and intraoperative blood loss, may all play roles in this response. ${ }^{12,13}$ According to a recent systematic review of vasospasm cases following pituitary surgery, $70 \%$ of pituitary adenoma resections developing vasospasm demonstrated the presence of significant SAH and/or intracranial hematoma with $23 \%$ requiring surgical evacuation. The most common presenting symptoms of these patients included changes in mental status $(56 \%)$ and motor deficits $(62 \%){ }^{14}$

Herein, we present a unique case of two subsequent strokes resulting from cerebral vasospasm occurring after giant pituitary adenoma resection without postoperative subarachnoid hemorrhage. While numerous reports of vasospasm occurring after transsphenoidal pituitary adenoma resection have been described, this case is unique in that no significant intraoperative blood loss or postoperative SAH on imaging occurred. Additionally, to our knowledge this is the only reported case of vasospasm occurring twice in the immediate and post-operative period after EES. This case reinforces the importance of maintaining a high-level of suspicion for acute vasospasm in patients who have recently undergone transsphenoidal pituitary resections regardless of the intraoperative blood loss and resulting blood products in the subarachnoid space.

\section{Case presentation}

\section{History and examination}

A 44-year-old, right-handed, morbidly obese $(\mathrm{BMI}=44.75)$ African-American female smoker initially presented with worsening daily frontal headaches, vision difficulties, nausea, and vomiting for 
four years prior to surgical intervention. Due to elevated prolactin levels, the patient was managed initially with bromocriptine therapy, which she eventually became unable to tolerate. After failing medical therapy and symptoms worsened, she was referred to neurosurgery. History and physical exam were otherwise unremarkable. Imaging revealed the presence of a giant pituitary adenoma (Figure 1). The decision was made to surgically resect the pituitary adenoma transsphenoidal ly through an endoscopic endonasal approach (EEA).

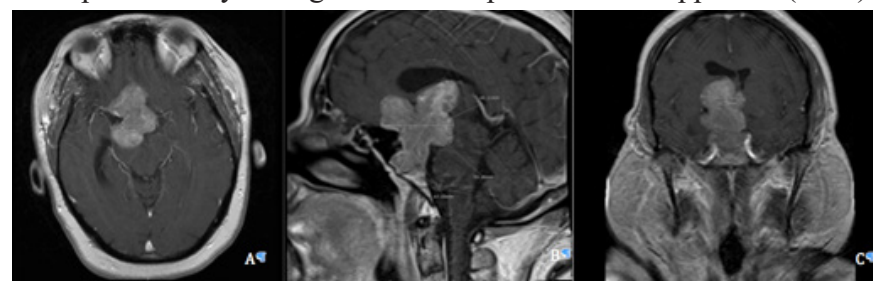

Figure I Pre-operative MRITI with contrast axial (A), sagittal (B), and coronal (C) views showing a large multilobular mass with lengthy extensions, vascular encasement, bone involvement, and mass effect.

In addition to a history of obesity and chronic smoking, the patient's comorbidities included congestive heart failure, malignant hypertension, rheumatoid arthritis, and diabetes mellitus type 2. Preoperative Electrocardiogram (ECG) revealed normal sinus rhythm with first-degree atrioventricular block and possible left atrial enlargement. Pre-operative routine serum pituitary panel revealed normal results with the exception of elevated prolactin levels at 85.9 $\mathrm{ng} / \mathrm{nL}$.

\section{Imaging}

Pre-operative magnetic resonance imaging (MRI) with and without contrast revealed a large mass arising from the pituitary gland with lengthy extensions, vascular encasement, bone involvement, mass effect causing optic nerves/chiasm compression, and hydrocephalus (Figure 1). The lobulated mass lesion arose from the sella with suprasellar extension predominantly towards the right, with measurements of $6.8 \times 5 \times 3.5 \mathrm{~cm}$ giving the impression of a giant pituitary adenoma. Lateral extension into the cavernous sinuses was also noted. The clivus and sphenoid sinuses also demonstrated tumor involvement. Mass effect upon the right lateral and third ventricle produced a midline shift and obstruction causing dilatation of the right lateral ventricle and left frontal horn.

Computed tomography angiography (CTA) showed no vessel stenosis or occlusion. A leftward displacement of the ACA vessels, a $3 \times 2 \times 3 \mathrm{~mm}$ anterior communicating artery aneurysm, and a hypoplastic right $\mathrm{A} 1$ segment anatomic variant were also noted.

\section{Operation}

Near total resection of the giant adenoma was achieved with EES Though there was some difficulty controlling bleeding due to the deep location, size, and vascular nature of the adenoma, blood loss was limited to $300 \mathrm{cc}$ requiring no transfusions. Successful hemostasis was achieved using copious warm water irrigation and bipolar coagulation. The superior part of the tumor did not descend during surgery demonstrating firm attachments to the surrounding anatomy and some residual tumor remained at the posterior aspect of the tumor bed. Reconstruction of the skull base defect was conducted with a vascularized nasoseptal flap. No other complications occurred during surgery.

\section{Pathological Examination}

Immunohistochemistry stains of the frozen section biopsy revealed a specimen positive for follicle stimulating hormone (FSH) and luteinizing hormone (LH) with a weak partial positive for prolactin and negative for p54 gene, thyroid stimulating hormone (TSH), growth hormone $(\mathrm{GH})$, and adrenocorticotropin hormone $(\mathrm{ACTH})$. Absent mitotic figures and a low proliferation index $(\mathrm{Ki} 67=1.7 \%)$ classified the tumor as a benign pituitary adenoma.

\section{Postoperative Course}

The patient was extubate immediately after surgery and had no new neurological deficits. The patient was transferred to the intensive care unit to appropriately control her malignant hypertension. Postoperative computed tomography (CT) head showed residual tumor but no intracranial hematoma, $\mathrm{SAH}$, pneumocephalus, or significant changes to baseline right ventricle dilatation. Follow-up MRI revealed blood products and intraventricular hemorrhage (IVH) as well as mass effect on the right cerebral peduncle but no signs of acute hemorrhage or injury of the hypothalamus.

On postoperative day 3 , the patient became agitated with some altered mental status and developed respiratory distress necessitating intubation. Pulmonary embolism workup was negative. An external ventricular drain (EVD) was placed to rule out hydrocephalus. CT of the head showed no evidence of acute hemorrhage but revealed small amounts of blood in the left occipital horn and an enlarging right ventricle. A fever of $101.5^{\circ} \mathrm{F}$ without signs of meningitis was noted. CSF, urine, blood, and sputum cultures were collected revealed growth in only sputum. Despite the negative cultures empiric antibiotics were started. Transcranial Doppler (TCD) revealed increased flow rate in cerebral vasculature indicating vasospasm as a possible cause of the patient's neurological decline.

On postoperative day 4, follow-up MRI continued to show no evidence of hemorrhage but revealed new ischemic areas in the posterior cortex paramidline apex and the right post central gyrus (Figure 2). 2D Echocardiogram was performed to rule out a cardiogenic etiology and revealed moderate to severe asymmetric septal hypertrophy, mild left ventricular diastolic dysfunction, and hyperdynamic left ventricular systolic function. CTA revealed scattered, multifocal stenosis of the bilateral M1 segments and of the left A1 segment confirming the diagnosis of vasospasm. Treatment with nimodipine and triple $\mathrm{H}$ therapy was immediately initiated. Due to persistent respiratory failure and prolonged ICU stay, the patient was tracheostomized.

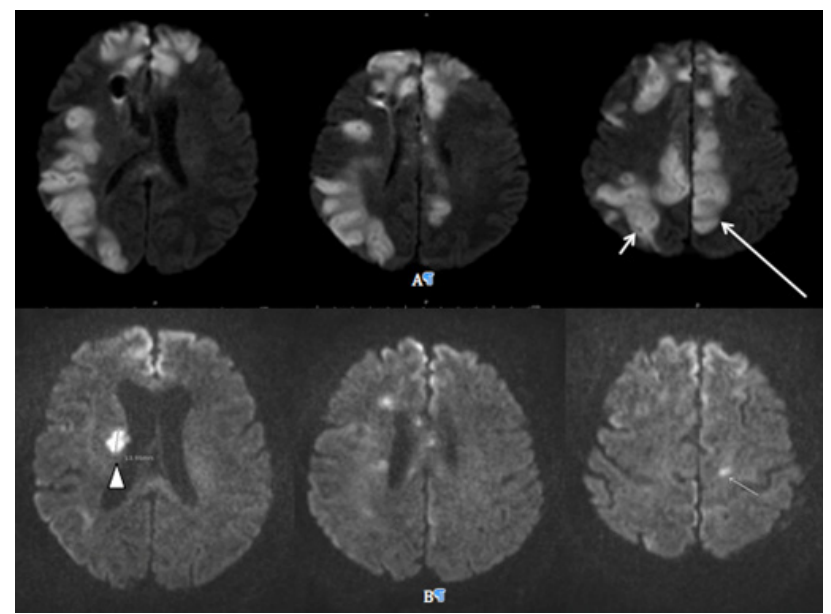

Figure 2 Postoperative day $4 \mathrm{MRI} D W I$ axial views (A) show ischemic areas in the posterior cortex paramidline apex (long arrow) and the in the right post central gyrus indicating an evolving stroke (short arrow). (B) Postoperative day 19 MRI DWI axial view demonstrating new infarct in area of right head of the caudate (arrowhead). 
Throughout the duration of hospital stay GCS ranged from $8 \mathrm{~T}$ to $11 \mathrm{~T}$. The patient continued to localize to pain with her bilateral upper extremities, but could only produce non-localizing movements of her lower extremities. The EVD was clamped and removed on postoperative day 12 without any reported CSF leakage or worsening ventriculomegaly and a percutaneous endoscopic gastrostomy tube was placed for nourishment. Serial CTs revealed no acute hemorrhage or other abnormal changes for the duration of the patient's management.

On postoperative day 19 a new area of infarct was noted on routine CT and MRI in the area of the right head of the caudate (Figure 2). No changes in the patient neurological exam were noted at this time. CTA again revealed no changes in the aforementioned aneurysm from baseline or any signs of occlusion and continued to reveal no acute hemorrhage.

Despite these new findings, diagnostic monitoring, and active treatments the patient's neurologic status remained unchanged. Once determined to be stable, the patient was discharged to a long-term rehabilitation facility.

\section{Discussion}

Herein, we present the only case to date involving two subsequent ischemic strokes, separated by 16 days, due to cerebral vasospasm status after EES for pituitary adenoma resection. Cerebral vasospasm after transsphenoidal pituitary surgery has been reported in a handful of cases and several conclusions have been made in regards to its etiologies, however, our case additionally highlights that vasospasm may still occur even in the absence of significant SAH and at dates later than the usual vasospasm window. While several key risk factors for vasospasm have been previously identified, it is important that a multifactorial approach be taken to identify and minimize these factors in the preoperative, operative, and postoperative setting in order to properly anticipate the risk for vasospasm. ${ }^{15}$

Preoperative evaluation is a crucial endeavor to assess for risks of surgical complications such as cerebral vasospasms and stroke. Pre-existing risk factors such as smoking history, chronic hypertension, diabetes mellitus, and obesity have all been identified as important risk factors for cerebral ischemic vasospasm. History of cigarette smoking has been demonstrated to be very important risk factor when considering the possibility of vasospasm after SAH. A recent retrospective study examining 370 patients with aneurysmal subarachnoid hemorrhage (aSAH) demonstrated that of current regular cigarette smokers 30\% developed symptomatic vasospasm compared to only $19 \%$ of nonsmokers in the postoperative period $(\mathrm{p}=0.0163) .{ }^{16}$ This association can be at least partially attributed to an increased concentration of endothelin after smoking occurs, which has been shown to be an important mediator in the vasospasm pathway. In addition to smoking, chronic hypertension has also been demonstrated to be an important risk factor for vasospasm most likely due to involved atherosclerosis. ${ }^{17}$ Diabetes and obesity have been shown to have a strong association with an increased risk of ischemic stroke both independently and synergistically., ${ }^{2,18,19}$

In addition to preoperative risk factors, various events during the surgical procedure are important to recognize when considering post-operative vasospasm risk. While efforts are usually made to limit manipulation of the relevant vascular, mechanical manipulation often does occur and is known to be an independent risk factor for development of clinically significant vasospasm. ${ }^{6,20}$ For this reason, it is important that adequate preoperative planning be conducted in order to understand the relevant anatomy of the local vasculature and minimize this mechanical irritation. ${ }^{21,22}$ While it is plausible that direct manipulation did occur in this case, it is unlikely as the superior aspect of the tumor capsule did not descend into the surgical field and was left attached to neurovascular structures.

In addition to vascular anatomy, it is important to acknowledge the anatomic proximity of the hypothalamus during preoperative planning as its location leaves it susceptible to damage. Damage to the hypothalamus has been shown to cause a release of chemical mediators into the CSF resulting in sympathetic stimulation influencing vasospasm. ${ }^{23}$ Had this injury occurred, the neurological sequelae would have been obvious in the immediately postoperative course; however, our patient was initially extubated without difficulty and had an otherwise uneventful recovery during the first three days following EES. Additionally, Surgical packing material used to repair the basal skull defect is also believed to play some role in eliciting vasospasm especially in cases of or excess packing. ${ }^{21}$ In our case, no fat graft or other material was used intradurally and all the reconstruction materials were placed extradurally thus making it unlikely to have produced any vascular irritation.

Special considerations in the postoperative period should include monitoring for accumulation of blood in the subarachnoid space and hematoma formation. Concern for the collection of blood products in the subarachnoid space cannot be understated as the degree of $\mathrm{SAH}$ is frequently considered the most important factor influencing vasospasm after aSAH. One recent prospective study demonstrated an average $\mathrm{SAH}$ volume of $17.67 \pm 5.18 \mathrm{ml}$ in patients who developed symptomatic vasospasm compared with $9.50 \pm 2.57 \mathrm{ml}$ in those not experiencing symptomatic or angiographic vasospasm..$^{24}$ In our case a small amount of IVH was observed, however, no identifiable subarachnoid hemorrhage was present. While, IVH has shown an association with vasospasm after aSAH it has not been previously been shown to have an association with vasospasm after pituitary surgery. ${ }^{25}$

The unique event of the second ischemic stroke from a nonthromboembolic etiology draws attention to the possibility of subsequent vasospasms after an initial vasospasm. Expected timing of vasospasm following pituitary surgery, or SAH, has been reported to occur in the range from hours to as a long as 13 days postoperatively, however, the second stroke in this case lies outside this range at 19 days. ${ }^{6}$ For this reason, neurosurgeons always should be cognizant and vigilant of this possibility.

\section{Conclusion}

Given this potentially devastating complication, it is vital that physicians be aware of this possible atypical presentation of cerebral vasospasm. Specifically, is important to understand that even in the absence of SAH other known risk factors such as smoking history, chronic hypertension, IVH, meningitis, and mechanical irritation may be enough to induce symptomatic cerebral vasospasm postoperatively. As research into improved prophylactic strategies continue to evolve, it is important for physicians to recognize known risk factors in order to minimize their influence and adequately anticipate potential complications.

\section{Conflicts of interest}

None.

\section{Permissions}

Figures were not obtained from elsewhere. 


\section{Acknowledgments}

None.

\section{References}

1. Abbott J, Kirkby GR. Acute visual loss and pituitary apoplexy after surgery. BMJ. 2004; 329(7459):218-219.

2. Wang F, Zhou T, Wei S, et al. Endoscopic endonasal transsphenoidal surgery of 1,166 pituitary adenomas. Surg endosc. 2015;29(6):1270-1280.

3. Black PM, Zervas NT, Candia GL. Incidence and management of complications of transsphenoidal operation for pituitary adenomas. Neurosurgery. 1987;20(6):920-924.

4. Barzaghi LR, Losa M, Giovanelli M, et al. Complications of transsphenoidal surgery in patients with pituitary adenoma: experience at a single centre. Acta neurochir. 2007;149(9):877-886.

5. Baggott CD, Aagaard-Kienitz B. Cerebral vasospasm. Neurosurg Clin N Am. 2014;25(3):497-528.

6. Aoki N, Origitano TC, al-Mefty O. Vasospasm after resection of skull base tumors. Acta neurochir . 1995;132(1-3):53-58.

7. Solenski NJ, Haley EC, Kassell NF, et al. Medical complications of aneurysmal subarachnoid hemorrhage: a report of the multicenter, cooperative aneurysm study. Participants of the Multicenter Cooperative Aneurysm Study. Crit care med. 1995;23(6):1007-1017.

8. Ropper AH, Zervas NT. Outcome 1 year after SAH from cerebra aneurysm: management morbidity, mortality, and functional status in 112 consecutive good-risk patients. J neurosurg. 1984;60(5):909-915.

9. Popugaev KA, Savin IA, Lubnin AU, et al. Unusual cause of cerebral vasospasm after pituitary surgery. Neurol Sci. 2011;32(4):673-680.

10. Barrow DL, Tindall GT. Loss of vision after transsphenoidal surgery. Neurosurgery. 1990;27(1):60-68.

11. Mawk JR. Vasospasm after pituitary surgery. $J$ neurosurg 1983;58(6):972.

12. Camp PE, Paxton HD, Buchan GC, et al. Vasospasm after transsphenoidal hypophysectomy. Neurosurgery. 1980;7(4):382-386.

13. Laws ER. Vascular complications of transsphenoidal surgery. Pituitary. 1999;2(2):163-170
14. Mansouri A, Fallah A, Cusimano MD, et al. Vasospasm post pituitary surgery: systematic review and 3 case presentations. Can J Neurol Sci. 2012;39(6):767-773

15. Puri AS, Zada G, Zarzour $\mathrm{H}$, et al. Cerebral vasospasm after transsphenoidal resection of pituitary macroadenomas: report of 3 cases and review of the literature. Neurosurgery. 2012;71(1 Suppl Operative): 173-180; discussion 180-181.

16. Inagawa $T$, Yahara $K$, Ohbayashi N. Risk factors associated with cerebral vasospasm following aneurysmal subarachnoid hemorrhage. Neurol Med Chir (Tokyo). 2014;54(6):465-473

17. Haak T, Jungmann E, Raab C, et al. Elevated endothelin-1 levels after cigarette smoking. Metabolism. 1994;43(3):267-269.

18. Andersen SS, Andersson C, Berger SM, et al. Impact of metabolic disorders on the relation between overweight/obesity and incident myocardial infarction and ischaemic stroke in fertile women: a nationwide cohort study. Clin Obes. 2015;5(3):127-135.

19. Olofindayo J, Peng H, Liu Y, et al. The interactive effect of diabetes and central obesity on stroke: a prospective cohort study of inner Mongolians. BMC neurol. 2015;15(1):1.

20. Kasliwal MK, Srivastava R, Sinha S, et al. Vasospasm after transsphenoidal pituitary surgery: a case report and review of the literature. Neurol India. 2008;56(1):81

21. Gupta R, Sharma A, Vaishya R, et al. Ischemic complications after pituitary surgery: a report of two cases. J Neurol Surg A Cent Eur Neurosurg. 2013;74(Suppl 1):e119-e123.

22. Bejjani GK, Sekhar LN, Yost AM, et al. Vasospasm after cranial base tumor resection: pathogenesis, diagnosis, and therapy. Surg Neurol. 1999;52(6):577-584.

23. Wilkins RH. Hypothalamic dysfunction and intracranial arterial spasms. Surg Neurol. 1975;4(5):472-480.

24. Jung SW, Lee CY, Yim MB. The relationship between subarachnoid hemorrhage volume and development of cerebral vasospasm. $J$ Cerebrovas Endovasc Neurosurg. 2012;14(3):186-191.

25. Kurschel S, Leber KA, Scarpatetti M, et al. Rare fatal vascular complication of transsphenoidal surgery. Acta neurochir. $2005 ; 147(3): 321-325$ 